This item was submitted to Loughborough's Research Repository by the author.

Items in Figshare are protected by copyright, with all rights reserved, unless otherwise indicated.

\title{
The real-time measurement of football aerodynamic loads under spinning conditions
}

PLEASE CITE THE PUBLISHED VERSION

https://doi.org/10.1177/1754337116677429

PUBLISHER

SAGE /@ IMechE

VERSION

AM (Accepted Manuscript)

\section{PUBLISHER STATEMENT}

This work is made available according to the conditions of the Creative Commons Attribution-NonCommercialNoDerivatives 4.0 International (CC BY-NC-ND 4.0) licence. Full details of this licence are available at: https://creativecommons.org/licenses/by-nc-nd/4.0/

\section{LICENCE}

CC BY-NC-ND 4.0

\section{REPOSITORY RECORD}

Passmore, Martin A., Simon Tuplin, and A. Stawski. 2016. "The Real-time Measurement of Football Aerodynamic Loads Under Spinning Conditions”. figshare. https://hdl.handle.net/2134/22969. 


\title{
The real-time measurement of football aerodynamic loads under spinning conditions.
}

\author{
Passmore M A, Tuplin S, Stawski A. \\ (accepted 11/10/2016 for inclusion in Journal of Sports Engineering and Technology)
}

\begin{abstract}
Aerodynamic effects play an important part in any sport where the ball experiences significant periods of free flight. This paper investigates the aerodynamic forces generated when a football is spinning quickly to generate swerve and more slowly to generate more erratic flight. The work reports on the application of an experimental method that measures the aerodynamic loads on a non-spinning, slowly spinning and fast spinning football, using a phase-locked technique so that orientation-dependent and steady 'Magnus' forces can both be determined.

The results demonstrate that the orientation-dependent aerodynamic loads, widely seen in non-spinning data in the literature, surprisingly persist up to the highest spin rates reported. When predicting ball flight, it is generally assumed that at low spin rates a quasi-static assumption is acceptable, whereby forces measured on a non-spinning ball, as a function of ball orientation, apply for the spinning case. Above an arbitrary spin rate, the quasi-static assumption is replaced with the assumption of a steady 'Magnus' force that is a function of spin rate and ball speed. Using a flight model, the quasi-static assumption is shown to be only applicable for the lowest spin rates tested and the assumption of a steady 'Magnus' force only applicable at the highest spin rates. In the intermediate spin rates (20 -40 rpm), the persistence of the orientation effects is shown to have sufficient effect on the flight to be an important additional consideration.
\end{abstract}

Keywords: Football, Soccer, Aerodynamics, Magnus, low spin, high spin, Spin Rate, Spin ratio, quasi-static, dynamic

\section{Introduction}

Aerodynamic effects play an important part in any sport where the ball experiences significant periods of free flight. There are many examples: football (soccer), tennis, baseball, golf, and cricket being some of the more popular, but there are numerous others. The initial impulse is imparted differently in each case: a kick, the impact from a racquet, the pitch or throw, impact from the club or the bowl. Whatever the source of the initial impulse, the participants seek to apply an accurate initial velocity and also a degree of spin to the ball. The purpose of the latter varies depending on the sport- in golf it can extend the range and improve accuracy; in football it can, for example, be employed to clear a defensive wall at a free kick- but 
often the spin is designed to deceive the opponent in some manner. The deception may be achieved by creating a large amount of swerve by applying a significant amount of spin to the ball, or alternatively by deliberately limiting the amount of spin, thereby generating unpredictable movement in the air. The third approach, relevant in some sports, is to use the spin to deceive the opponent after the ball has bounced.

The focus of this paper is on the behaviour through the air, and in particular, on understanding the forces generated when the ball is spinning quickly to generate swerve and more slowly to generate an erratic flight. The work reported investigates this using a new experimental approach that measures the aerodynamic loads on a non-spinning, slowly spinning and fast spinning ball continuously such that the different effects present can be identified.

\section{Background}

At any instant during a ball's flight, an aerodynamic load acts on the ball that is the integrated effect of the pressure and shear stress distributions. For convenience in ball aerodynamics the integrated load is separated into the drag and lateral force. The drag force is the resistance to motion through the air and the dominant contribution to it comes from the pressure drag, caused by the formation of a large turbulent wake behind the ball following separation. The lateral force is also caused primarily by an imbalance of the pressure distribution, but in the lateral sense (strictly normal to the spin axis), and it can result in a deflection in the path of the ball in the direction of the lowest pressure region. The lateral force acts normal to the freestream velocity and the spin axis for a spinning ball.

Under situations where the ball is spinning, the effect of the rotation is normally to advance the separation point on the reverse rotating side and to delay it on the opposite, generating a lateral momentum exchange 1,2. This is commonly referred to as the Magnus effect and the lateral force is related to the ball speed, spin rate and ball diameter. The effect has been investigated in a number of papers including for smooth spheres ${ }^{3}$, for baseballs ${ }^{4,5}$, golf balls ${ }^{6,7}$ and for footballs ${ }^{8,9}$. A 'reverse Magnus' effect is also possible when the combination of spin rate and ball speed produces a laminar separation on one side of the ball and a turbulent on the other. Reverse Magnus effects have been demonstrated in measurements by Maccoll ${ }^{3}$ and Passmore ${ }^{10}$.

For non-spinning balls, the asymmetric flow fields required to generate lateral forces may arise through differences in surface roughness or features on opposing sides of the ball. The work of Achenbach ${ }^{11,12}$, though not dealing specifically with asymmetry, provides useful insight, as it shows the effects of both Reynolds number and (uniform) surface roughness on the separation angle. In particular, Achenbach shows the ability of the flow to stay attached further around the sphere with both increasing Reynolds number and increasing roughness. The consequence for ball flight is that when a ball has different degrees 
of roughness on opposing sides, the separation angle is different and a lateral force is generated. This effect is particularly important in sports such as cricket and baseball, but Passmore ${ }^{9}$ showed that footballs also generate orientation-dependent lateral forces that are attributed to asymmetric separation caused by the seams at the edges of each panel. The asymmetric separation was confirmed by Ruck ${ }^{13}$ in a series of flow-field measurements conducted using PIV where he shows that the lateral force is correlated with the separation angle. Passmore ${ }^{9}$ also demonstrates, using a flight model, that if you assume quasi-static conditions, then when a football is rotating slowly, the changes in the lateral force caused by this asymmetric separation will cause 'knuckling' of a football such as is also used to great effect in baseball when the ball is pitched with almost no rotation.

The final mechanism for generating lateral forces arises from the natural unsteadiness associated with bluff bodies. This unsteadiness arises, in part, because of small movements in the separation line and can be particularly pronounced when the separation is not fixed by a sharp edge, but rather, located in the region of a radius ${ }^{14}$; as balls are spheres, the separation is always on a radius and the wake inherently unsteady. Passmore ${ }^{10}$ explores the effect by measuring the unsteady force for a non-spinning football and superimposing the fluctuating load onto an otherwise steady lateral force in a simulated flight. The maximum differences in lateral position during the flight (with /without fluctuating component) are quoted as $\pm 5 \mathrm{~mm}$. Goff ${ }^{15}$ also measures the fluctuating load for a non-spinning football and plots lift against side-force (orthogonal components of lateral force) showing the difference in variation of the force for two footballs tested in two orientations. The fluctuating forces that Goff ${ }^{15}$ reports are large compared to those reported by Passmore ${ }^{10}$ and are likely a consequence of mounting the football directly to the outside of the panel rather than employing a rigid mount as described by Passmore ${ }^{9}$. When mounted to the panels, the stiffness is relatively low and varies depending on the location on the panel and the orientation of the underlying carcass (a stitched fabric structure between the bladder and the outer panels). In the authors' experience, this gives rise to a coupling of the unsteady forces to the deflection of the ball and hence unreliable measurement of the unsteady loads. In this paper, the focus is on the spinning phenomenon rather than the unsteadiness.

The two in-flight scenarios of slow and high spin rates are usually treated quite separately, both during measurement and in any subsequent analysis. For high spin conditions and a given ball, the 'Magnus' force is assumed to be a function of the ball speed and spin rate, and essentially the flow field is considered to be steady. The wind tunnel measurements are typically sampled over a number of seconds and averaged for each set spin rate and tunnel speed, as performed in Passmore ${ }^{9}$, for example. For low spin conditions, a quasi-static assumption is applied and the flight calculated from statically acquired data. For example, Passmore ${ }^{8,9}$ acquires the non-spinning data by performing a yaw sweep between $\pm 90^{\circ}$ in $5^{\circ}$ steps sampling at $100 \mathrm{~Hz}$ and averaging over 30 seconds for each point. 
In order to calculate flight trajectories, Passmore ${ }^{9}$ makes the quasi-static assumption that the forces measured for a non-spinning ball exist when the ball is spinning, and that this applies up to a maximum spin rate of $10 \mathrm{rpm}$, above which the orientation data is replaced with time-averaged spinning data (Magnus force). It is unlikely however that such a simplification of the two scenarios is realistic. This work therefore concentrates on a new method for obtaining the aerodynamic force data on spinning balls. The objective is to identify whether the two regimes are realistic and, if they are, what the appropriate maximum spin rate is for application of the quasi-static assumption.

\section{Experimental Methods}

All tests were conducted in the Loughborough University wind tunnel. The facility is open circuit with a closed working section of $1.32 \times 1.9 \mathrm{~m}$. The maximum working speed of $45 \mathrm{~m} / \mathrm{s}$ is comfortably above the maximum for a free kick of $34 \mathrm{~m} / \mathrm{s}$ reported by Neilson ${ }^{17}$. The working section turbulence intensity is approximately $0.2 \%$ and spatial uniformity $\pm 0.2 \%$ of mean velocity. For further details on the tunnel, see Johl ${ }^{18}$.

The lateral force and drag data is measured using a purpose built overhead balance with an integrated motor that provides the orientation and spin capability. Data can be acquired continuously while the ball is spinning to give an output of lateral force and angular orientation. Closed loop speed control is via an integrated encoder; and when in spinning mode, the angular position of the ball is calculated from a once per revolution marker combined with the measured spin rate. To ensure accurate control of the spin rate, the PID control was tuned with the ball mounted as in the tests. To check on the stability of the control, the spin rate was recorded over a 60 second period for spin rates between 4 and $240 \mathrm{rpm}$; the standard deviation for each sample lay between 0.046 and $0.068 \mathrm{rpm}$. The balance can also be used in a non-spin mode, where the motor is essentially used as a conventional yaw drive to provide orientation.

To facilitate mounting on the shaft, the footballs are filled with a two-part polyurethane expandable foam which is then drilled to accommodate a $20 \mathrm{~mm}$ diameter shaft. While the foam is curing, the ball is held in a mould to ensure the correct diameter and sphericity. Thus the ball is rigidly mounted and has the added advantage that the footballs all have the same diameter. As a control for this process, the balls are measured on a CMM machine pre- and post-fill for diameter and sphericity, with additional measurements of the surface texture and seam profile taken. Typically there is to be less than a $+/-0.5$ $\mathrm{mm}$ deviation from the target diameter of $219 \mathrm{~mm}$, a mean change of seam depth of 150 microns and no increase of sphericity. Surface texture is largely unaffected by the fill process. The spin-balance is equipped with a collet to take the $20 \mathrm{~mm}$ diameter ball mounting shaft.

The ball support effects are determined by measuring the loads on the support only. To ensure that both the direct forces and the ball interference with the support are measured, a ball is held in proximity to the support using a second support, such that the ball is in the correct location but with a small clearance so that the support-only forces are measured. So when a support from below is used, the tare is determined 
by supporting the ball from behind; and when the tare for the support from behind is measured, the ball is supported from above. The coefficients are calculated by subtracting the ball support forces and applying equations 1 and 2 .

$$
C_{d}=\frac{D}{\frac{1}{2} \rho A v_{\infty}^{2}} \quad \text { (1) } \quad C_{\text {Lat }}=\frac{F_{\text {Lat }}}{\frac{1}{2} \rho A v_{\infty}^{2}}
$$

During initial commissioning tests, the ball spin rate and maximum test speed were found to be limited due to the unsteady nature of the aerodynamic forces overloading the sensitive integrated spin balance. A number of steps were taken to resolve this. Initially, for convenience, the support shaft designed for use with the underfloor balance had been used in the spin balance. However, this was unnecessarily long so was shortened by $135 \mathrm{~mm}$ to reduce the moments applied at the balance load cells and increase the resonant frequencies of the combined system of the ball support and balance so that they were not excited by the aerodynamic fluctuations. The reduction in length also positions the ball the same distance from the tunnel roof as it is from the tunnel floor when mounted to the underfloor balance (ball centre $330 \mathrm{~mm}$ from the boundary). As a further step, the support shaft was enclosed within a NACA 0021 aerofoil section machined from model board with sufficient internal clearance to ensure that the ball can spin freely. The arrangement is illustrated in Figure 1. The addition of the shroud further reduced any vibration from the system, allowing high-quality measurements up to an airspeed of $30 \mathrm{~m} / \mathrm{s}$.

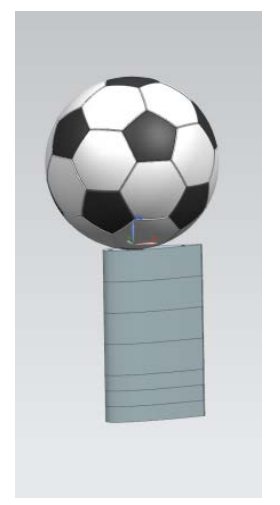

(a)

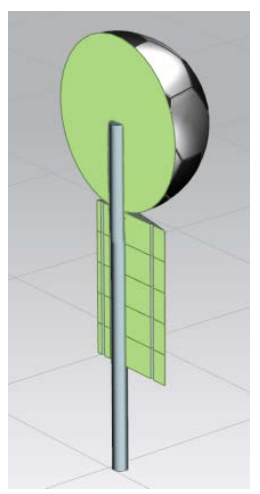

(b)

Figure 1- Illustration of the aerofoil shroud (a) and mounting internal arrangement (b)

Data is acquired from the spin balance at a fixed frequency of $300 \mathrm{~Hz}$ and processed using a 'binning' method, where each data point is assigned to a bin according to the associated angular orientation. Every data set is the average of 20 complete rotations of the ball. This averaging approach ensures that the 
effects of ball orientation are captured clearly but separated from the inherent unsteadiness. The effect of changing bin size on the lateral coefficient is illustrated in Figure 2. The smallest bin size of one degree shows a small amount of noise and the largest of $15^{\circ}$ shows some smearing of the data. For the results presented in this paper, a bin size of $5^{\circ}$ is used throughout. A consequence of the fixed sample rate is that the number of data points in any particular bin depends on the spin rate. At the lowest spin rates presented ( $4 \mathrm{rpm}$ ), each $5^{\circ}$ bin is the average of 1333 data points; and at the highest spin rate (90 rpm), each bin contains 55 data points.

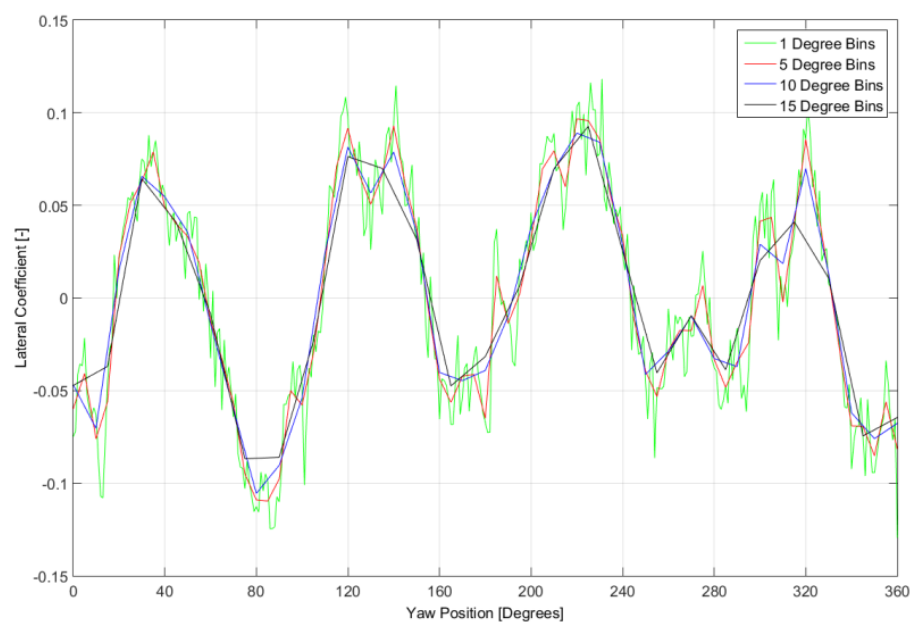

Figure 2 - Lateral coefficient against orientation for varying bin size - spinning test.

In the sections that follow, results are presented for two ball types: the adidas Tango12, used in the 2012 European championships, and the adidas Brazuka, used in the 2014 world cup. Images of the two balls are shown in Figure 3.

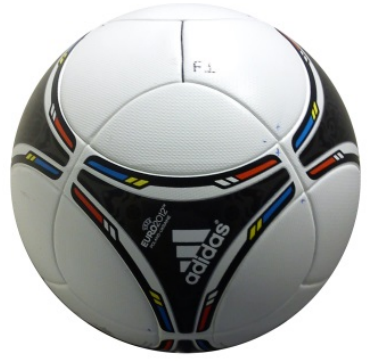

(a)

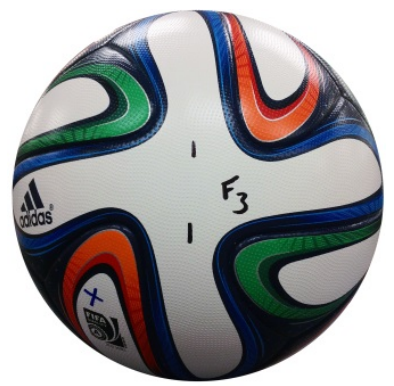

(b)

Figure 3 - Images of the test balls, adidas Tango12 (a) and adidas Brazuka (b). 


\section{Results and Discussion}

\section{Non-spinning tests- Ball One - Brazuka}

Figure 4 shows drag coefficient as a function of Reynolds number for a single ball orientation using the support from below shielded with the NACA aerofoil and also with the support from behind through the wake in the configuration typically used to capture the drag. Using the support from behind, the result is as expected ${ }^{10}$ with a post-critical drag coefficient below 0.2 . When supported from below, the postcritical drag coefficient is approximately 0.4; this is a little lower than that generally found when the ball is supported from beneath ${ }^{10}$ and is attributed to the influence of the aerofoil section reducing the effect of the support and modifying the wake. With the ball supported from behind, the transition region occurs at a ball speed of between approximately 12 and $16 \mathrm{~m} / \mathrm{s}$; but when supported from below, the transition is more gradual, with the post critical drag reached at a speed of about $14 \mathrm{~m} / \mathrm{s}$. It is worth noting that while the results are quite different in the two mounting positions, it is not possible to conclude that the mount from behind gives a more representative drag coefficient. The support from below disrupts the wake locally, changing the rear pressures and the drag. Equally, devices situated within the wake of a bluff body (for example, cylinders, small plates or splitters) are sometimes employed to stabilise the wake and increase rear pressure, thereby reducing drag, suggesting that mounting from behind may produce an artificially low drag coefficient. In the flight simulations reported later in the paper, the average drag coefficient for the ball supported from behind and from below is used. This follows the recommendation of Tuplin et al ${ }^{19}$ who show that the drag coefficient has little practical effect on causing player deception.

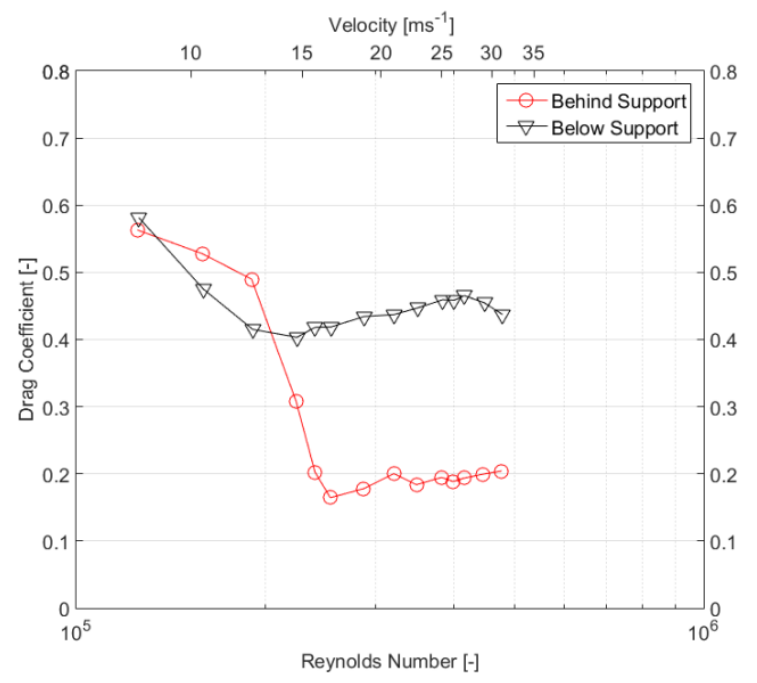

Figure 4 - Drag and lateral coefficient as a function of Reynolds number.

Figure 5 shows the Lateral coefficient against ball orientation at 10, 15, 20 and $25 \mathrm{~m} / \mathrm{s}$ (Re No. 1.56x10 to $3.95 \times 10^{5}$ ). The results show a significant degree of Reynolds sensitivity with changes in both the 
amplitude and the overall shape of the plots. The largest lateral coefficients are seen at the lowest test speed (and Reynolds number) where the ball is in the sub-critical region; here the peak-to-peak variation in the lateral coefficient is approximately \pm 0.4 . At $15 \mathrm{~m} / \mathrm{s}$ and above, the tests are in the post-critical region and the lateral coefficients are considerably smaller with a range of approximately \pm 0.1 at the highest test speed of $25 \mathrm{~m} / \mathrm{s}$. The shape of the plots show considerable variation for the four Reynolds numbers tested. At the lowest and highest Reynolds numbers, the pattern is similar, showing four clear 'cycles' that reflect the number of panels used to construct this ball. At the intermediate Reynolds numbers, the pattern is less clear, so may not be so easily attributed to the panel arrangement; however, the lateral coefficients are quite small, suggesting less orientation sensitivity in general. The mean lateral coefficient, also shown on the plot is close to zero in all cases.

It is likely that the support also has some effect on the lateral coefficients. This has not been directly investigated here; however, the key objective of this work is to identify whether the orientation-dependent forces measured for a non-spinning ball persist when the ball is spinning. The comparison required for this is unaffected by the test methodology even if the forces are not identical to those found in free flight. It is also worthy of note that the largest lateral forces produced are sufficient to generate realistic simulated flights and to indicate that the support is not suppressing the wake deflection to a significant extent. This is consistent with a turbulent separation where only a small portion of the support fairing is immersed in the wake.
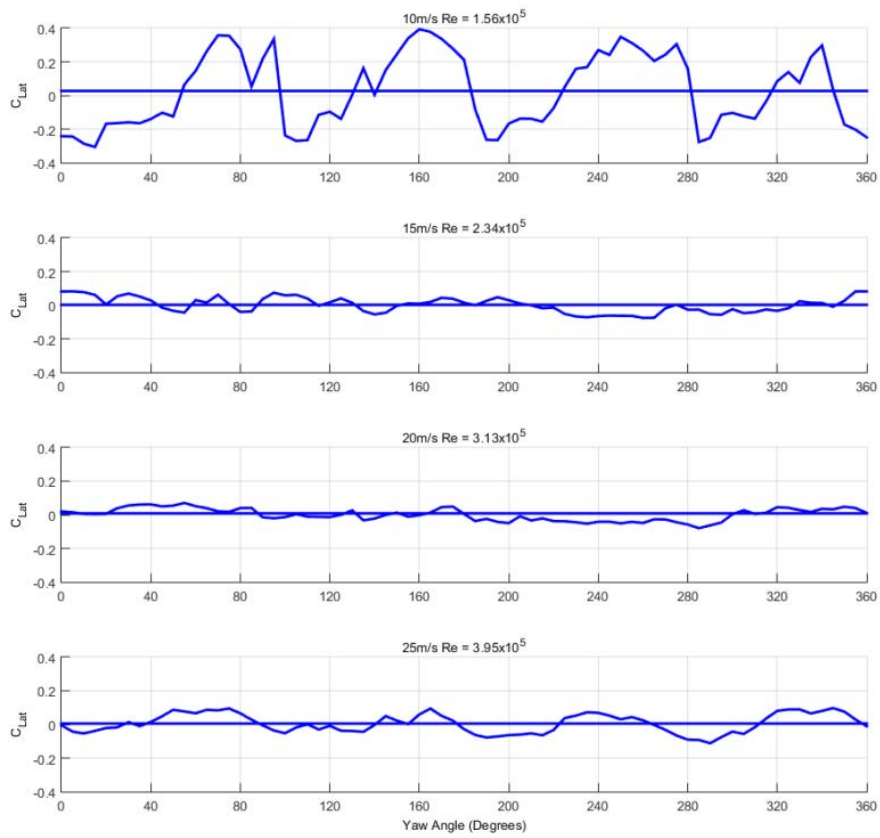

Figure 5 -Lateral coefficient against yaw orientation -Brazuka non-spinning test. 


\section{Spinning tests- Ball one - Brazuka}

The spinning experiment included tests for all four Reynolds numbers shown in the non-spinning tests, but full results are included only for the highest and lowest Re No (equivalent to $25 \mathrm{~m} / \mathrm{s}$ and $10 \mathrm{~m} / \mathrm{s}$ ). Considering the data collected at $25 \mathrm{~m} / \mathrm{s}$ and shown in Figure 6, each plot is at a constant spin rate and shows the lateral coefficient as a function of orientation measured dynamically and compared with the static orientation data repeated from Figure 5. The spin rate ranges from 4 rpm (spin ratio 0.0018) to 90 rpm (spin ratio 0.0415). Note that spin ratio is defined as the ratio of the ball surface tangential velocity to the ball speed $\omega r / U$ where $\omega$ is the spin rate in rads/sec, $r$ the ball radius $(\mathrm{m})$ and $U(\mathrm{~m} / \mathrm{s})$ the test or ball speed. At the lowest spin rate (4 rpm), the dynamic data matches the static very closely, suggesting that the quasi-static assumption is appropriate. As the spin rate is increased, the dynamic data is seen to shift, such that the mean lateral force is no longer zero. This is the Magnus effect and is the force typically measured in a time-averaged spinning test and it increases progressively with spin rate to 0.038 at $40 \mathrm{rpm}$ and 0.098 at $90 \mathrm{rpm}$. However, the dependence on orientation, while progressively reducing in amplitude, does not disappear from the data. Even at the highest spin rate (90 rpm), there is still considerable variation in the lateral coefficient as a function of orientation, of order \pm 0.07 . This is still about $70 \%$ of the range measured in the static case. Furthermore, throughout the range of spin rates, the orientation dependence remains well correlated to the static yaw data. This effect of a shift in the mean lateral coefficient as the Magnus force increases with spin rate and the continued dependence on orientation is repeated for the tests at $20 \mathrm{~m} / \mathrm{s}$ and $15 \mathrm{~m} / \mathrm{s}$ (not shown).
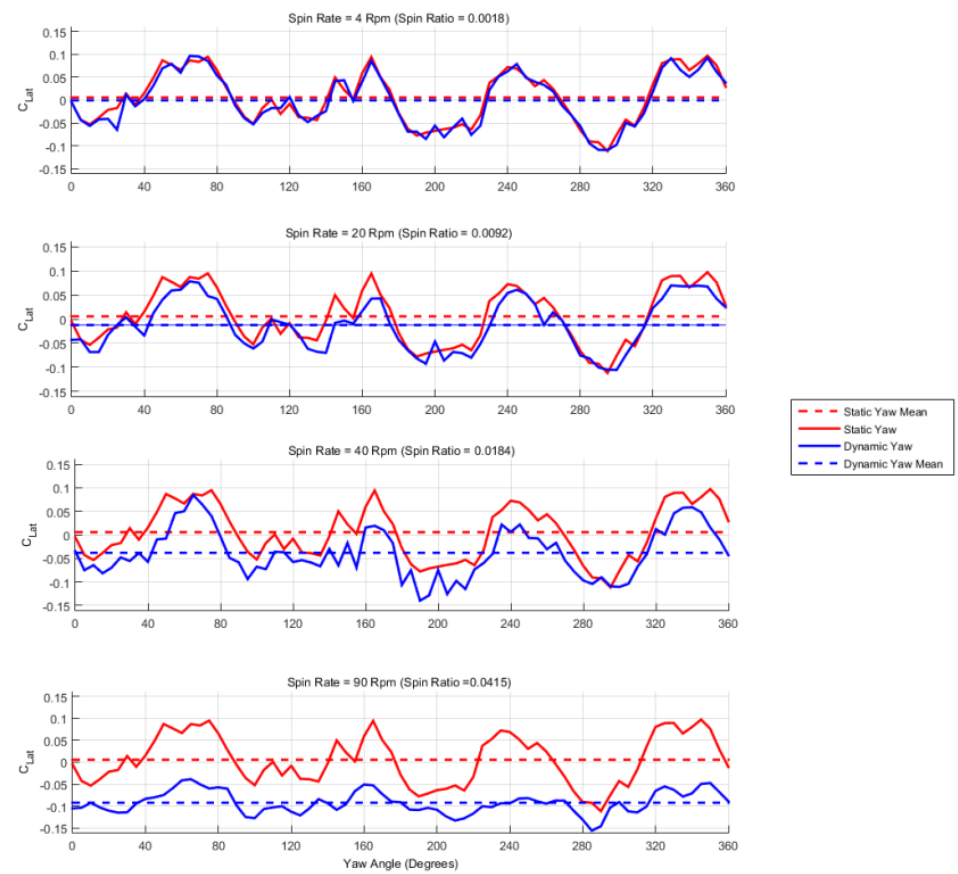

Figure 6 - Lateral coefficient against orientation - Brazuka spinning ball at $25 \mathrm{~m} / \mathrm{s}$ 
Figure 7 presents similar results for the lowest test speed of $10 \mathrm{~m} / \mathrm{s}$, again for spin rates ranging from 4 rpm (spin ratio 0.0018) to $90 \mathrm{rpm}$ (spin ratio 0.0415). Again at the lowest spin rate, the quasi-static assumption appears to be a reasonable approach, although the match between the dynamically measured and static data is not quite as consistent as seen in the post-critical data presented in Figure 6. As the spin rate is increased, the sensitivity to orientation reduces rapidly and there is only a small change in the mean lateral coefficient. At a spin rate of $40 \mathrm{rpm}$, the peak-to-peak variation with orientation has reduced to approximately $30 \%$ of that seen in the static result, and at $90 \mathrm{rpm}$ it has reduced further to only $15 \%$.

Although the mean lateral coefficients are quite small, they are worthy of report; at the lowest spin rate it is a small positive value, 0.021 , similar to the static value so essentially capturing a small degree of asymmetry. As the spin rate increases, the mean lateral coefficient reduces to zero at $20 \mathrm{rpm}$ and -0.007 at $40 \mathrm{rpm}$ and then returns to a positive value of 0.018 at $90 \mathrm{rpm}$. This suggests reverse Magnus effects that arise when the low ball speeds are combined with certain spin rates. They are consistent with that seen in Passmore ${ }^{10}$ in conventional time-averaged spin data.
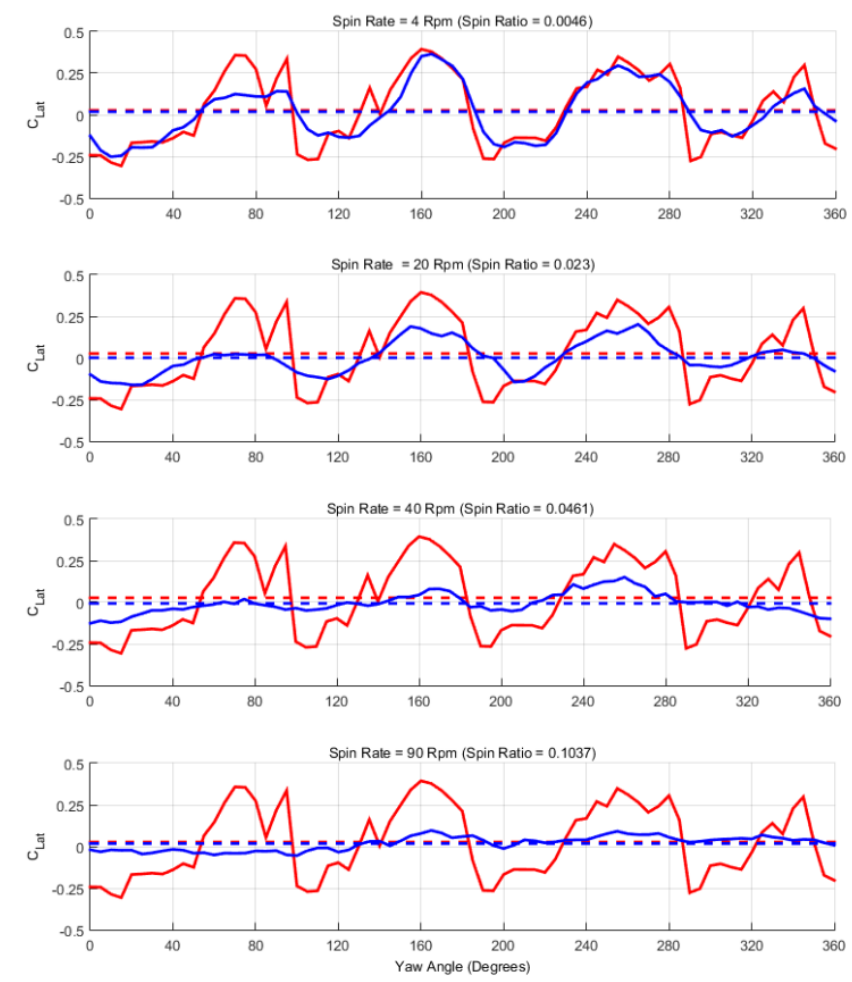

Figure 7 - Lateral coefficient against orientation - Brazuka spinning ball at $10 \mathrm{~m} / \mathrm{s}$ 


\section{Spinning tests - Ball two - Tango12}

For the purposes of providing some comparison and assessing whether the results for the Brazuka are repeated for a second ball, the adidas Tango12 was tested. The results for the test at $25 \mathrm{~m} / \mathrm{s}$ are summarised in Figure 8. Because this ball has a different panel configuration, the characteristic is quite different to the Brazuka data seen in Figure 6 and Figure 7, but the conclusions from the static and spinning tests are essentially the same. At the lowest spin rate, the dynamic data matches the static well and as the spin rate is increased, the mean lateral coefficient increases, reaching 0.062 at $90 \mathrm{rpm}$. The orientation sensitivity remains as the spin rate increases such that at $90 \mathrm{rpm}$ the peak-to-peak variation is still about $90 \%$ of that seen in the static test. The underlying shape of the plot is also preserved, although less well than for the Brazuka.
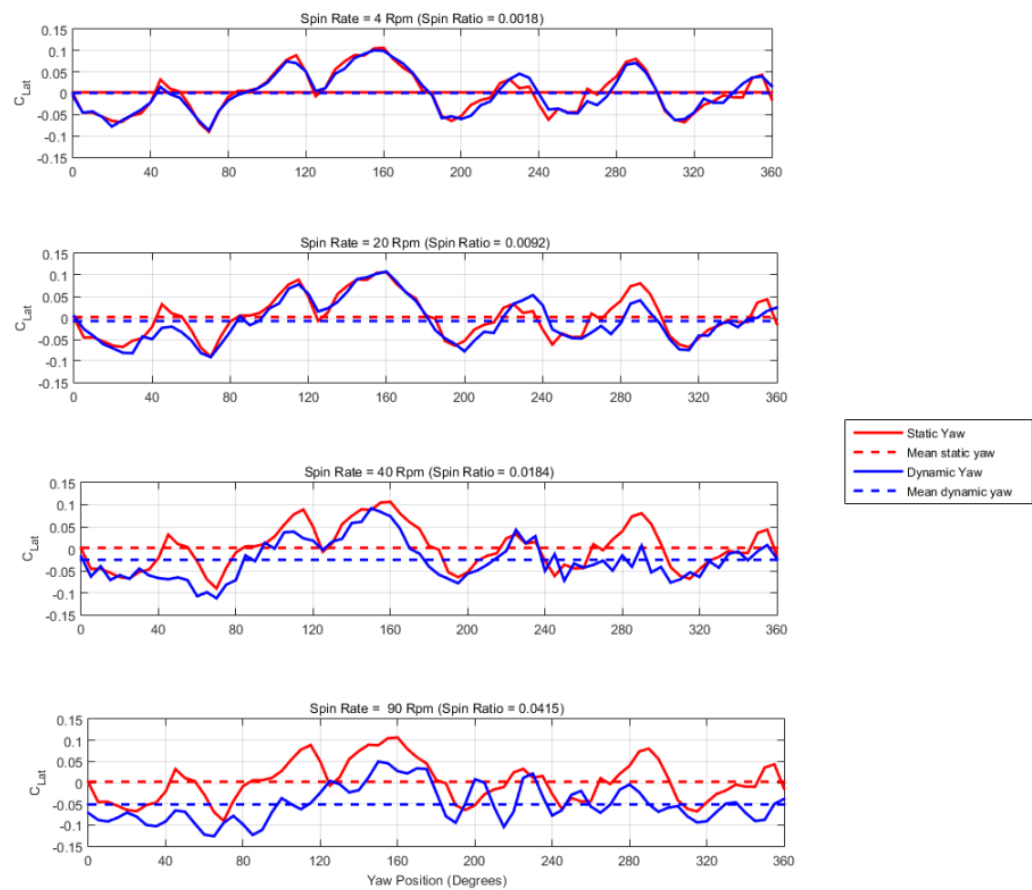

Figure 8 - Lateral coefficient against orientation - Tango12 at 25 m/s

\section{Comparison of mean lateral forces.}

Figure 9 shows the mean lateral coefficients for both balls plotted against spin ratio. The plots show quite different results for the two balls with the Tango12 generally producing less lateral force for the same ball speed and spin ratio. This is consistent with the findings of Passmore ${ }^{10}$ that showed a considerable range of performance for five different FIFA approved balls. It is also noted that both balls show reverse 
Magnus at low speed $\left(\operatorname{Re}=1.56 \times 10^{5}\right)$ over a range of spin ratios, but at the higher Reynolds numbers, this occurs only at very low spin ratios.

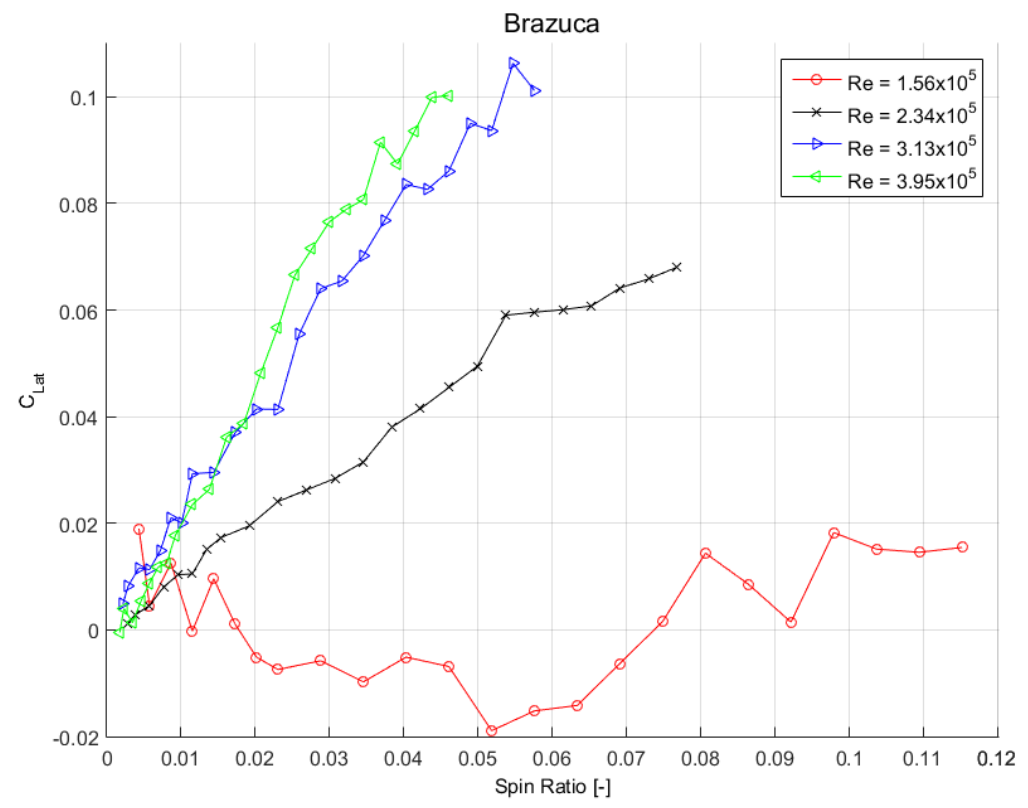

Figure 9 - Mean lateral coefficients against spin ratio. Brazuka and Tango12.

\section{Discussion}

The results clearly capture both the orientation effects responsible for knuckling seen in all footballs ${ }^{10}$ and the Magnus forces used to generate swerve. What is unexpected is the persistence of the orientationdependent lateral forces up to quite high spin rates, showing that the assumption that the two phenomena can be measured and analysed separately requires closer examination. If the lateral coefficients are considered, for example Figure 6 showing the behaviour of the Brazuka at a speed of $25 \mathrm{~m} / \mathrm{s}$, the quasistatic assumption looks reasonable at $4 \mathrm{rpm}$, but by $20 \mathrm{rpm}$, both orientation and Magnus effects are present. At the higher spin rates, the Magnus force looks likely to dominate but the persistence of the orientation sensitivity may also contribute to the flight path. At the lower test speed (10 m/s, Figure 7), the mean lateral forces appear too small at all spin rates to be significant, but the orientation effects again look significant even at the highest spin rate reported. Similar comments are also appropriate for the second test ball (Tango12, Figure 8). The natural conclusion is that both effects must be measured simultaneously to get a complete picture of the aerodynamic loads, using a method such has been described here. However, considering the forces in isolation, while interesting, is essentially subjective because it is the effect of the forces on the flight of the ball under realistic conditions that is important. In 
practice, a comparison of flights must also be treated with caution. For example, Goff ${ }^{15}$ calculates the flight paths for two balls with the same initial conditions and draws conclusions based on the difference between the two flights. However, this approach assumes that humans use a computation-based approach to determine the future ball path, but Craig ${ }^{20}$ points out that the human interception process is characterised by a reliance on prospective information carried in the perceptual flow. In other words, the players track the ball continuously throughout the flight, updating their action continuously, because they cannot directly predict the future path. In the analysis of ball flights, the unpredictable flights are therefore only those where significant changes in path occur rapidly enough that the player is unable to react, or where the player observes rapid changes that prevent appropriate updates to the interception strategy.

Figure 10 shows a comparison of a high spin (90 rpm) flight simulated using only the mean lateral force, i.e. the Magnus force, compared directly with a flight simulated using the full dynamically measured aerodynamic forces as described above. The flights are generated using the flight model described by Tuplin ${ }^{19}$.
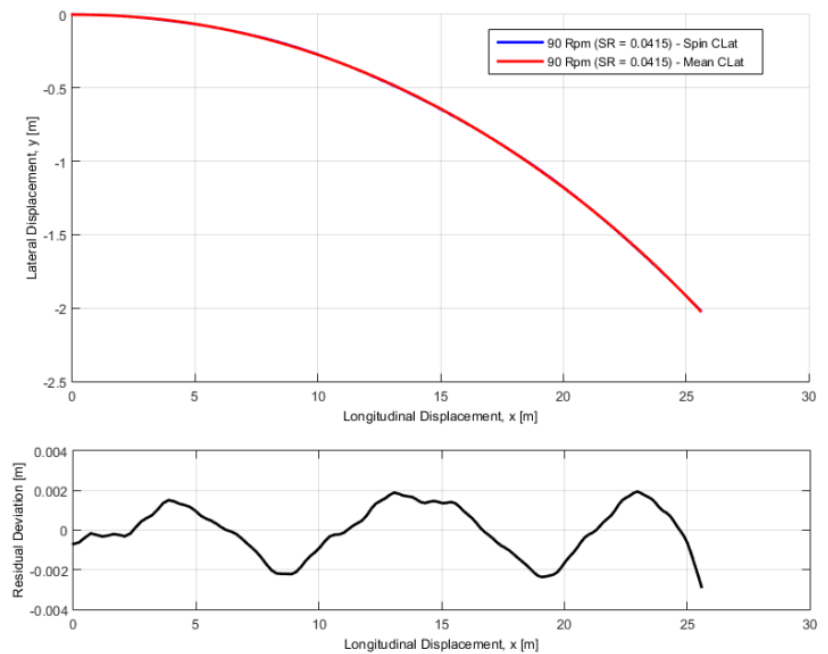

Figure 10 - Comparison of flight trajectories using mean and full orientation-dependent data. (a) flight path, (b) difference in paths. (Launch conditions: $25 \mathrm{~m} / \mathrm{s}, 1^{\circ}, 90 \mathrm{rpm}$, vertical spin axis.

Figure 10(a) shows that the two flight paths overlay each other to the extent that only one path is clearly visible, so Figure 10(b) is included to show the small difference in lateral deviation for the two simulations. The contribution from the orientation effect is shown to be less than $\pm 3 \mathrm{~mm}$ and is well below any threshold that is likely to be perceived by the players or to cause any deception. In this regard, it may be concluded that under high-spin conditions, the assumption of a constant spin 'Magnus' force is reasonable. This is however a single flight, so a more in-depth look at a range of flights is shown in 
Figure 11 and Figure 12. In Figure 11, each plot shows four flight speeds for a fixed value of spin rate and a single start orientation. In Figure 12, each plot shows a number of flights with different start orientations for a single value of spin rate and a single ball speed of $25 \mathrm{~m} / \mathrm{s}$.

The launch angle, of 30 degrees, is the same for every flight in these two figures; and because the simulation stops when the ball strikes the ground, the duration of the flight and distance travelled reduces with reducing launch velocity. This is an important consideration often overlooked when making assessments of ball performance based only on the forces because a variation in the aerodynamic coefficients will not always result in perceptibly unpredictable behaviour. For example, a ball travelling at $10 \mathrm{~m} / \mathrm{s}$ (sub-critical region) may exhibit relatively high lateral coefficients, but the consequence is mitigated by the low speed and the fact that the length of the flight in both time and distance is limited by the physics. This is illustrated in Figure 11 . At $10 \mathrm{~m} / \mathrm{s}$, the ball travels only 5 metres in about $0.5 \mathrm{~s}$ before contacting the ground again. The lateral deviation is unlikely to deceive a receiving player because the flight is slow and the ball only moves one way during the flight. At the higher spin rates in Figure 11 $(b, c)$, the evidence that orientation is influencing the trajectory is still clear well into the region where the Magnus effect might normally be assumed to dominate. However, the lateral movement due to orientation is practically quite small; at $20 \mathrm{rpm}$ a maximum of approximately $18 \mathrm{~mm}$ at $15 \mathrm{~m} / \mathrm{s}$ and $\pm 20 \mathrm{~mm}$ at $25 \mathrm{~m} / \mathrm{s}$ where the Magnus force is seen to be the primary effect. At the highest spin rate shown in Figure 11(d), $60 \mathrm{rpm}$, the flights appear predictable and dominated by the Magnus effect.

The interesting behaviour at low spin is captured in Figure 12a , where, with the fixed initial velocity of $25 \mathrm{~m} / \mathrm{s}$, the path depends on the start orientation and the curvature changes during flight; this is something that cannot be anticipated by the receiving player and here the lateral deviations are considerable. This is consistent with a quasi-static assumption and is behaviour demonstrated, to varying degrees, for all balls ${ }^{10}$.

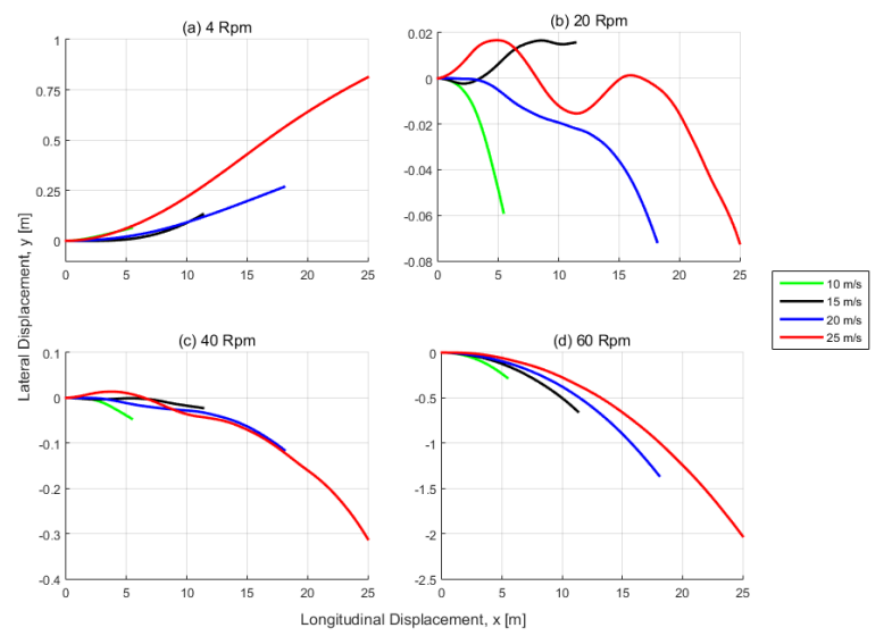

Figure 11 - Influence of ball velocity and spin rate on flight trajectory. (Launch conditions: $\mathbf{1 8}^{\circ}$, vertical spin axis, initial velocity and rpm as indicated in figure) 


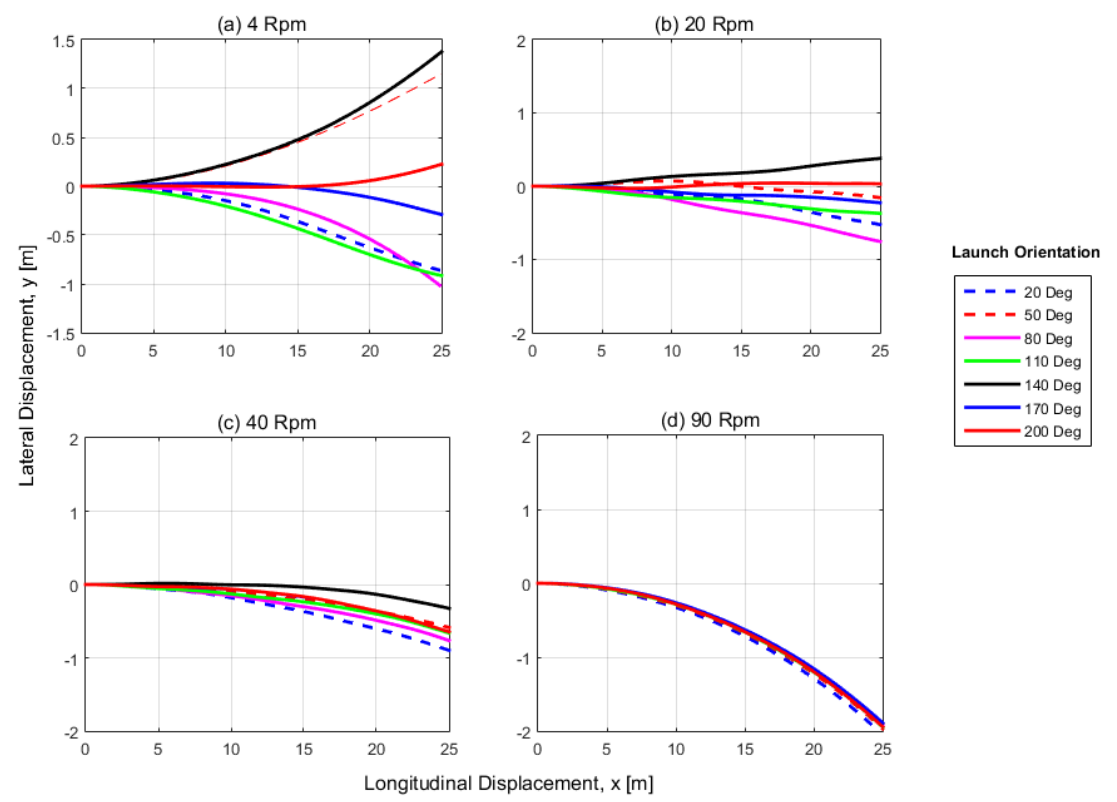

Figure 12 - Influence of initial ball orientation on flight trajectory (Launch conditions: $25 \mathrm{~m} / \mathrm{s}, \mathbf{1 8}^{\circ}$, vertical spin axis, $\mathbf{r p m}$ as indicated in figure).

Further insight is provided by considering the progression in spin rate illustrated in Figure 12. At the lowest spin rate (4 rpm), the quasi-static assumption is reasonable. At $20 \mathrm{rpm}$, there is still considerable variation in the flight depending on the start position; but more importantly, the changes of direction associated with the changing orientation through the flight are visible in the trajectory. At 40 rpm, there is variation depending on the initial orientation, but the flights are strongly biased to one direction by the Magnus force and any knuckling is not clearly evident. Even at 90 rpm there is still some difference between the flights, although an assumption that the flights are determined by the Magnus force alone is reasonable. For this case, the difference, $20 \mathrm{~m}$ into the flight, in lateral displacement between the ball moving most and least is about $100 \mathrm{~mm}$, or half a ball diameter. This may appear significant, but a player interception model based on the continuous (imperfect) tracking and update of the flight path would suggest that problems for the players occur only when the ball is subject to changes in curvature of the flight. So at these high spin rates, such difficulties are unlikely to arise.

The region of most interest is therefore when the spin rate is of order 20 - $40 \mathrm{rpm}$ with ball speed of approximately $15 \mathrm{~m} / \mathrm{s}$ or greater. In this range, it is possible to generate sufficient unpredictability that there is the potential to deceive an opponent. Interestingly, at the higher end of this spin rate range, Figure 12c, the difference in lateral deviation between the ball moving the least and most is almost $0.6 \mathrm{~m}$; but this in itself does not necessarily lead to deception. It is only if the remaining orientation sensitivity within each flight is enough to cause perceptible and unpredictable changes in direction that the receiving player may be deceived. Such variation exists, of course, for a single ball and all balls. 


\section{Conclusions}

- A technique for measuring the orientation-dependent aerodynamic forces on a spinning football has been developed by applying a phase-locked technique to measurements from a specialist wind tunnel balance with an integrated spin capability. The results presented are a unique insight into the in-flight aerodynamic loads.

- The results demonstrate that the orientation-dependent aerodynamic loads, widely seen in static data in the literature, persist up to high spin rates. At the highest spin rates described (90 rpm), the orientation-dependent variation in the lateral coefficients is still as much as $70-90 \%$ of that seen in the static case.

- The orientation-dependent lateral coefficients for a non-spinning ball have zero mean when averaged over a complete revolution, but under spinning conditions are superimposed onto a non-zero mean lateral force that is widely described as the Magnus force.

- At the lowest spin rates used, the dynamic data is shown to match the static orientation data well, providing confidence in the measurement and processing techniques employed.

- When predicting ball flight, it is generally assumed that at low spin rates, a quasi-static assumption is acceptable; whereby statically measured forces that are a function of ball orientation apply, but that above this low threshold, an assumption of a steady 'Magnus' force applies. Using a flight model, the quasi-static assumption is shown to be only applicable for the lowest spin rates tested but also that the assumption of a steady 'Magnus' force is only reasonable at the higher spin rates investigated. In the intermediate spin rates (20 -40 rpm), the persistence of the orientation effects is shown to have sufficient effect on the flight to be an important additional consideration.

\section{References}

1. Mehta, R.D., Aerodynamics of Sports Balls, Ann. Rev. Fluid Mech. Annual Reviews inc. 1985

2. Mehta, R.D., Pallis, J.M., The aerodynamics of a tennis ball, Sports Engineering, V4, pp177 - 189, 2001.

3. Maccoll, J.W., Aerodynamics of a Spinning Sphere, Glasgow University, 1928.

4. Briggs, L.J. Effect of spin and speed on the lateral deflection of a baseball; and the Magnus effect for smooth spheres. Am. J. Phys., 27, 589, 1959.

5. Watts, R.G., Ferrer, R., The lateral force on a spinning sphere: Aerodynamics of a curveball, American Journal of Physics, V55 (1) pp40-44, 1987.

6. Davies, J.M., The Aerodynamics of Golf Balls, Journal of Applied Physics, V20(9) pp821-828, 1949. 
7. Bearman, P.W., Harvey, J.K., Golf Ball Aerodynamics, Aeronautical Quarterly, May 1976

8. Carré, M. J.; Asai, T.; Akatsuka, T.; Haake, S. J. (2002). The curve kick of a football 2: flight through the air. Sports Engineering, 5, Vol. 1, pp 183-192

9. Passmore, M.; Spencer, A.; Tuplin, S.; Jones, R. (2008). Experimental studies of the aerodynamics of spinning and stationary footballs. Proceedings of IMechE, Part C. Vol. 222, pp 195-205.

10. Passmore, M.; Rogers, D.; Tuplin, S.; Harland, A. (2011). The aerodynamic performance of a range of FIFA approved footballs: Proc. IMechE Vol 226 Part P: Journal of Sports Engineering and Technology.

11. Achenbach, E., Experiments on the flow past spheres at very high Reynolds numbers, Journal of Fluid Mechanics, V54(3) pp565-575, 1972

12. Achenbach, E., The effects of surface roughness and tunnel blockage on the flow past spheres, Journal of Fluid Mechanics, V65(1) pp113-125, 1974

13. Ruck, S., Spencer, A., Passmore, M.A., The influence of football surface characteristics on flow separation angle. Fachtagung, Lasermethoden in der Stromungsmesstechnik, Sep 2011, Ilmenau.

14. Fuller, J,. Passmore, M.A. The importance of rear pillar geometry on fastback wake structures. Journal of Wind Engineering and Industrial Aerodynamics, 125, 2014 pp 111-120

15. Goff J E, Asai, T., Hong, S., A Comparison of Jabulani and Brazuca non-spin aerodynamics. 2014, Proc. IMechE Part P: Journal of Sports Engineering and Technology.

16. Carre, M J, Goodwill, S.R., Haake, S.J., Understanding the effect of seams on the aerodynamics of an association football. 2004, Proc. IMechE Vol 219, PartC: Journal of Mechanical Engineering Science.

17. Neilson, P.J., Jones, R. An exact method for the sphericity measurement of soccer balls, Proceedings of the Institution of Mechanical Engineers, Part B: Journal of Engineering Manufacturing, V217(B5), pp715 - 719, 2003

18. Johl, G., Passmore, M.A., Render, P., The Design Methodology and Performance of an Indraft Wind Tunnel, The Aeronautical Journal, V108 (1087), pp. 465-473. 2004.

19. Tuplin, S., Passmore, M.A., Rogers, D., Harland, A.R., Lucas, T., Holmes, C., The application of simulation to the understanding of football flight. Proceeding of the Institution of Mechanical Engineers, Part P, Journal of Sports Engineering and Technology, June 2012, Vol 220 No 2 pp 134142.

20. Craig, CM,. Berton, E,. Rao, G,. Fernandez, L,. Bootsma, R J,. Judging where a ball will go: the case of curved free kicks in football. Naturwissenschaften, 2006, 93: 97-101 